\title{
Transcatheter closure of patent foramen ovale using the Amplatzer septal occluder to prevent recurrence of neurological decompression illness in divers
}

K P Walsh, P T Wilmshurst, W L Morrison

\begin{abstract}
Objective-Large flap valve patent foramens may cause paradoxical thromboembolism and neurological decompression illness in divers. The ability of a self expanding Nitinol wire mesh device (Amplatzer septal occluder) to produce complete closure of the patent foramen ovale was assessed.

Patients-Seven adults, aged 18-60 years, who had experienced neurological decompression illness related to diving. Six appeared to have a normal atrial septum on transthoracic echocardiography, while one was found to have an aneurysm of the interatrial septum.

Methods-Right atrial angiography was performed to delineate the morphology of the right to left shunt. The defects were sized bidirectionally with a precalibrated balloon filled with dilute contrast. The largest balloon diameter that could be repeatedly passed across the septum was used to select the occlusion device diameter. Devices were introduced through 7 F long sheaths. All patients underwent transthoracic contrast echocardiography one month after the implant.
\end{abstract}

Results-Device placement was successful in all patients. Device sizes ranged from 9-14 $\mathrm{mm}$. The patient with an aneurysm of the interatrial septum had three defects, which were closed with two devices. Right atrial angiography showed complete immediate closure in all patients. Median (range) fluoroscopy time was 13.7 (6-35) minutes. Follow up contrast echocardiography showed no right to left shunting in six of seven patients and the passage of a few bubbles in one patient. All patients have been allowed to return to diving. Conclusion-The Amplatzer septal occluder can close the large flap valve patent foramen ovale in divers who have experienced neurological decompression illness. Interatrial septal aneurysms with multiple defects may require more than one device. (Heart 1999;81:257-261)

Keywords: patent foramen ovale; decompression illness; Amplatzer septal occluder

The atrial septum's oval fossa is patent in one quarter of adults. ${ }^{1}$ In most cases this represents probe patency, such that a pathologist's probe or a cardiologists catheter can barely be passed through. However, in a smaller percentage there is wider patency (pencil patency) ${ }^{2}$; a larger area of the flap valve is non-adherent to the limbus of the oval fossa, and a finger or inflated rubber balloon can be advanced backwards and forwards across the septum. It is probable that patients with a larger rim of the flap valve non-adherent are at risk of paradoxical embolus resulting in cryptogenic stroke ${ }^{3-7}$ or neurological decompression illness in divers. $^{8}$

Patency of the foramen ovale in life is usually diagnosed by contrast echocardiography with or without Valsalva manoeuvre. ${ }^{4}$ Transoesophageal echocardiography may also be helpful, and may show left to right and right to left shunting across the foramen ovale on colour flow mapping, in addition to bubble passage during contrast injection. ${ }^{57}$ If the patent foramen ovale is thought to be the mechanism for the neurological symptoms then closure may be indicated. ${ }^{9}{ }^{10}$ However, since patency is relatively common, false positive diagnosis is possible in a patient in whom a probe patent foramen is coincidentally associated with a neurological event. ${ }^{11}$ Methods to discriminate the size of a patent foramen ovale are based on the intensity of left atrial opacification during contrast injection with either transthoracic or transoesophageal echocardiography. ${ }^{7}$ In divers, certain types of decompression illness are related to presence of large right to left shunts on contrast echocardiography. ${ }^{812} 13$

Transcatheter closure of the patent foramen ovale to prevent paradoxical embolism has already been described using either the clamshell device ${ }^{9}$ or the adjustable buttoned device. ${ }^{14} 15$ The clamshell device was withdrawn from clinical trials because of an $83 \%$ rate of leg fractures, ${ }^{16}$ while the adjustable buttoned device remains in use but is cumbersome to implant. The Amplatzer septal occluder (AGA Medical, Minnesota, USA) has been developed to occlude secundum atrial septal defects with left to right shunts. ${ }^{17}{ }^{18}$ We describe the use of the Amplatzer septal occluder in patients with presumed paradoxical embolism. We also looked at the angiographic demonstration and balloon sizing of the patent foramen ovale in our group of patients.

Patients and methods

Seven adults (one female), aged 18-60 years, were referred for transcatheter closure of atrial 
level right to left shunts on transthoracic contrast echocardiography. After diving, each had experienced symptoms and signs of shunt related decompression illness. Three divers were professional and four were amateurs. Six divers had neurological decompression illness (spinal in three, cerebral in three) on one occasion. The seventh diver had two separate episodes of cerebral decompression illness. Onset of symptoms was within 30 minutes of surfacing. Their dive profiles followed decompression algorithms currently used in Britain and generally considered to be safe. One also had cutaneous decompression illness. One man (38 years old) also had a history of a small cerebrovascular accident unrelated to diving, with complete recovery within four days. Investigations at the time of his stroke revealed no possible cause other than a patent foramen ovale. In all seven divers, lung function tests and chest radiographs were normal, excluding pulmonary disease known to predispose to decompression illness. ${ }^{13}$ Six appeared to have a normal atrial septum on transthoracic echocardiography while one was found to have an aneurysm of the interatrial septum. In each, with a single contrast injection, a large right to left shunt was seen soon after right heart opacification during normal quiet respiration, without provocative manoeuvres such as cough or Valsalva.

AMPLATZER SEPTAL OCCLUDER

The Amplatzer septal occluder is constructed from 0.004 or 0.005 inch Nitinol wires, tightly woven into two interconnected saucer shaped discs. The cylindrical connection between the two discs serves to stent the defect and its diameter is considered to be the size of the occluder. The left atrial disc is slightly larger than the right atrial one and extends $5 \mathrm{~mm}$ beyond the cylindrical component. There is a thin polyester patch sewn into each of the three components of the device, which serve to promote thrombosis and occlusion within the device. A delivery cable is attached to the device by a screw at the proximal end of the device. Before introduction the device is collapsed into a tubular loader by pulling on the delivery cable.

The district ethics committee approved use of the device, which now has a "CE" mark (Conseil d'Europe) for atrial septal defect closure. Patients gave informed consent before implantation.

\section{PROCEDURE}

All closures were performed under general anaesthesia to allow continuous multiplane transoesophageal imaging of the atrial septum and related structures. A 7 French $(F)$ gauge sheath was placed in the right femoral vein and a smaller sheath in the left femoral artery for pressure monitoring. The right heart was catheterised to check whether there was a left to right shunt and to measure the pulmonary artery pressure. A biplane right atriogram in $30^{\circ}$ right anterior oblique and $45^{\circ}$ left anterior oblique with $25^{\circ}$ cranial angulation projections, with the catheter pointing towards the fossa ovalis, was performed to delineate the angiographic morphology of the right to left shunt (fig 1).

A sizing balloon (Robicsek, BVM Medical, Leicester, UK), inflated with a range of volumes of dilute contrast, was calibrated with a template. A multipurpose catheter was then passed across the defect and into the left upper or lower pulmonary vein to allow an exchange length guidewire to be placed. The sizing balloon was then passed over the exchange guidewire and inflated with dilute contrast. The volume that could be passed, with minor resistance, from both left to right and right to left was noted. This was used as the stretched diameter of the defect and a similar diameter occluding device was chosen. A $7 \mathrm{~F}$ long sheath and dilator were passed across the atrial septum over the exchange guidewire used for balloon sizing. The dilator and wire were removed. The appropriately selected device was screwed on to the delivery wire and compressed by withdrawing it into the loader. The compressed device was then introduced into the sheath and advanced across the atrial septum to the end of the long sheath. The left atrial retaining disc was then extruded. The extruded disc and introducer sheath were then withdrawn as a single unit until resistance to further withdrawal was felt, which should be at the fossa ovalis. While maintaining constant tension on the delivery wire the introducer sheath was peeled back to expand the rest of the occluder. The position of the occluder was checked with transoesophageal echocardiography before release. Care was taken to ensure the device did not protrude into the superior vena cava or right upper pulmonary vein. The device was released by anticlockwise rotation of the delivery cable.

The patient with an aneurysm of the interatrial septum required a longer procedure during which two devices were implanted, as two of the three defects were widely separated (fig 2). An additional $8 \mathrm{~F}$ venous sheath was placed in order to allow the defects to be sized separately. In this patient the devices were implanted sequentially and both devices remained attached to their respective delivery cables until they were shown to line up on the atrial septum without device overlap.

Patients received prophylactic antibiotics to cover the implant procedure and for six months after implantation. Echocardiography with colour flow mapping was performed before discharge to ensure maintenance of position. They also received aspirin $300 \mathrm{mg}$ per day for three months in order to prevent excessive thrombus formation on the device. Follow up contrast echocardiography was performed one month after the implant. This involved a set protocol in which six contrast injections were given using coughing and Valsalva manoeuvres to promote right to left shunting across any residual communication. ${ }^{12}$

\section{Results}

All patients had devices implanted successfully. There were no acute complications. The 

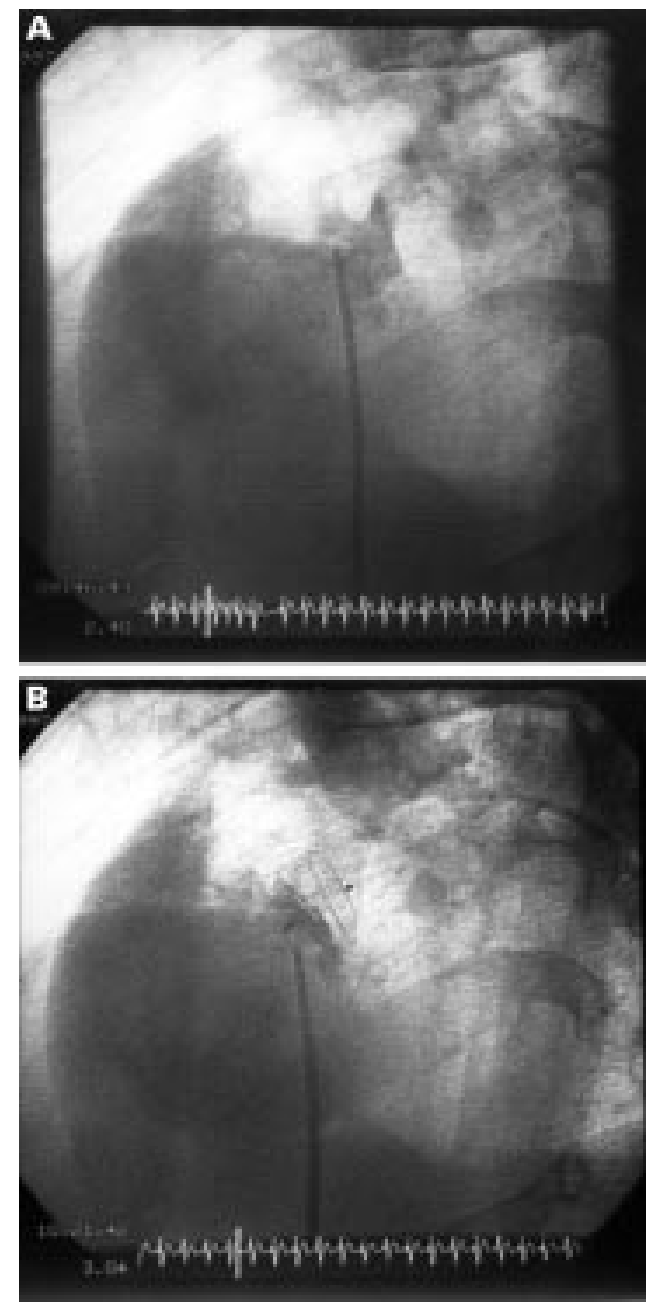

Figure 1 (A) Right atrial angiogram with the catheter pointing towards the foramen ovale, showing passage of contrast across the foramen into the left atrium. (B) After placement of a $9 \mathrm{~mm}$ Amplatzer septal occluder there is complete angiographic occlusion of the foramen ovale.

procedure time ranged from $60-180$ minutes and the fluoroscopy time from 9-45 minutes.

On the basis of transoesophageal echocardiography, right atrial angiography, and balloon sizing, six patients had flap valve patent foramen ovales and the other had an interatrial septal aneurysm with multiple septal defects. This patient was a professional diver and he had three separate defects in his aneurysmal atrial septum requiring two separate devices ( 9 and $10 \mathrm{~mm}$, respectively) for complete closure. His third defect was close to the lower second device; although it appeared open at the end of the procedure it had closed by the time follow up transoesophageal echocardiography was performed after one month.

The six patients with flap valve patent foramens required occluders of between 10 and $14 \mathrm{~mm}$. The occluders all appeared to be in the superior part of the interatrial septum near the superior vena cava. None produced any turbulence in the superior vena caval flow. Right atrial angiography showed complete occlusion in all patients (fig 1). Colour flow transthoracic echocardiography before discharge showed some left to right shunting in three patients and complete occlusion in the others. Contrast echocardiography with Valsalva manoeuvre was performed one month after implantation and showed complete occlusion in six patients and a trivial right to left shunt in the patient who received a $14 \mathrm{~mm}$ device. In that case between one and 10 bubbles were seen in the left heart when contrast injection was supplemented with the Valsalva manoeuvre on three occasions, but no shunt was detected on three other occasions. Transoesophageal echocardiography performed in the patient with an interatrial septal aneurysm and three defects requiring two devices showed complete occlusion of all three defects (fig 2).

Formal anticoagulation with warfarin, even in the patient who had had a previous stroke, was not advised because of a lack of evidence for the effectiveness of any post device closure anticoagulation protocol. However, it may be prudent to consider short term anticoagulation for patients who have had previous strokes.

All patients have been allowed to return to diving six weeks after the implant. There has been no further neurological decompression illness in any of the patients over a 3-12 month follow up period.
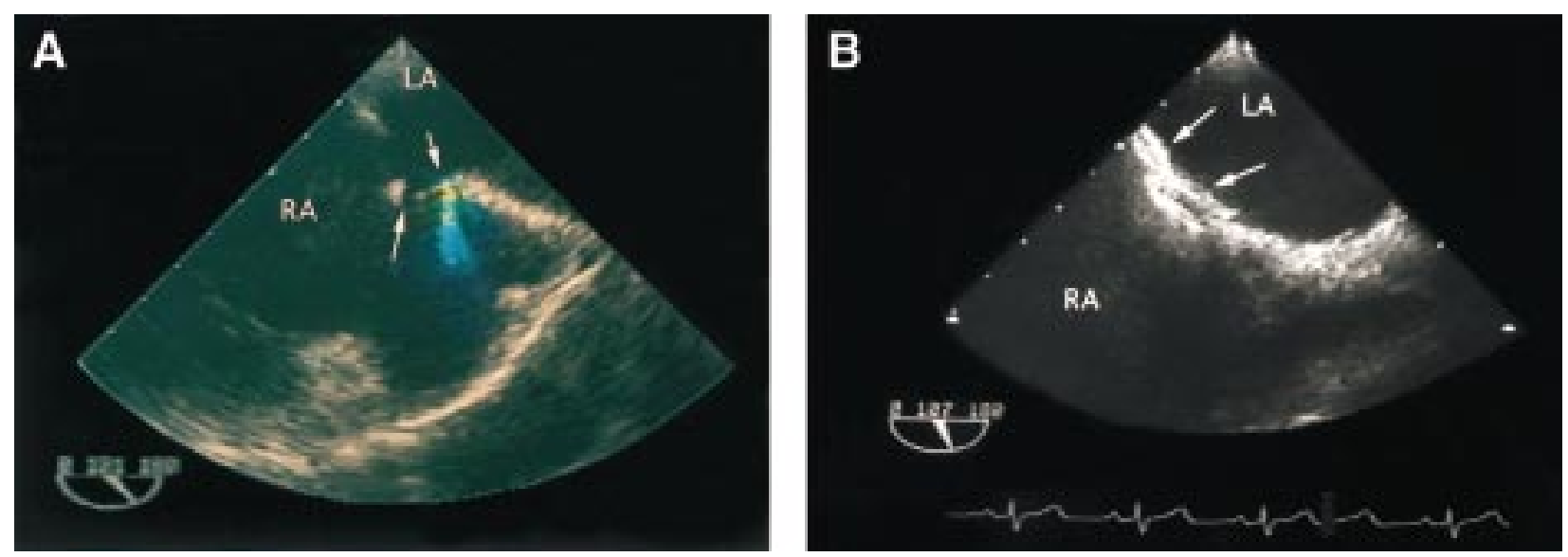

Figure 2 (A) Transoesophageal echocardiogram of the patient with an aneurysm of the interatrial septum. The aneurysm bulges into the right atrium and two small defects (indicated by the arrows) are identified in this longitudinal plane. A left to right colour jet can be seen passing through the upper defect. (B) Two Amplatzer septal occluders have been placed. The arrows indicate each of the devices, which are aligned sequentially on the atrial septum. There was no residual flow on contrast echocardiography. $L A$, left atrium; $R A$, right atrium. 


\section{Discussion}

The patent foramen ovale and aneurysmal interatrial septum are being increasingly incriminated in cerebral thromboembolism and neurological decompression illness. Approximately a quarter of adults have a patent foramen; most of these are probe patent and probably do not cause any harm, as they are too small, with a serpiginous course through the interatrial septum, to permit significant paradoxical thromboembolism. It is possible for microbubbles to pass across such defects but they are few in numbers and require repeated injections to demonstrate. In contrast, we believe that flap valve foramens are much more likely to allow significant paradoxical thromboembolism. Flap valve foramens are easy to demonstrate on contrast echocardiography with or without Valsalva manoeuvres. Right atrial angiography shows that the defects relate to the lack of adherence of the septum primum to the superior part of the limbus of the oval fossa. A 10-14 mm diameter balloon could be passed backwards and forwards across the flap valve foramen in our patients.

In most patent foramen ovales the right to left sizing is said to be larger than the left to right. The fact that the divers' left to right and right to left stretched diameters were similar may indicate that this select group of patients had larger non-adherent flap valves than usually found. There is no information on the incidence or consequences of such flap valve foramens in the general adult population, or in patients with cryptogenic stroke or neurological decompression illness. However, our observations suggest that these large flap valve foramens can be most easily recognised by the presence of a large right to left shunt at rest without provocative manoeuvres. This contrast echocardiographic appearance is present in about $5 \%$ of the population. ${ }^{13}$ Clearly balloon sizing and right atrial angiography will usually only be done at the time of planned transcatheter closure. However, if the defect was only demonstrated to be probe patent then it is possible that device closure would not be helpful and that there is another cause for the symptoms.

Technically it was very easy to close the patent foramen ovale with this device. The device stents the potential space between the limbus and the septum primum. Thrombus and later fibrous tissue ingrowth into the device and endothelialisation of the discs accomplish occlusion. ${ }^{18}$ Because the potential defect is stented, there was transient left to right shunting seen in some cases on echocardiography the following day. However, at one month there was neither left to right nor right to left shunting in six of the seven divers. We have previously described the use of the inverted adjustable buttoned device to occlude the patent foramen ovale in divers with neurological decompression illness. ${ }^{19}$ The Amplatzer septal occluder was much easier to use because of its one part introduction method, rapid release by a screw mechanism, and facility to retrieve and reposition as required. The adjustable buttoned device requires a two part introduction method, and release is achieved by pulling a double loop of nylon thread out as a single strand. The adjustable buttoned device is retrieved by reinforcement of the loading wire with a snare; once retrieved it cannot be reused.

It is possible that a non-self centring device would have managed to close the interatrial septal aneurysm with multiple defects more easily. ${ }^{14}$ However, the new version of the clamshell device (CardioSEAL; Nitinol Medical Technologies, Boston, Massachusetts, USA) has already experienced a significant although considerably lower rate of device arm fracture $^{20}$ and requires an $11 \mathrm{~F}$ delivery sheath. The ASDOS device (Osypka, Grenzach-Whylen, Germany) ${ }^{21}$ and the Sideris (Custom Medical Devices, Athens, Greece) adjustable buttoned device require a two part introduction method and are more cumbersome to implant. The Das Angel Wings device (Microvena Corp, Minnesota, USA) is also self centring but requires an $11 \mathrm{~F}$ delivery system for the size that might be required to close a flap valve foramen. Also the sharp corners on this device have been reported to erode the aorta, causing a delayed tamponade in a patient having an atrial septal defect closure. ${ }^{22}$ This device has since been modified to round the corners to prevent aortic erosion.

Our study group comprised divers who had experienced neurological bends, which we have attributed to a large patent foramen ovale. Three were professional divers and four were keen amateurs. We justified implantation of devices in the three professional divers, who would otherwise not have come to early medical attention but for their diving, by the fact that successful device implantation has allowed them to continue with their livelihood. Justification in the keen amateurs was based on their strong desire to continue diving and the long term brain scan changes seen in some divers. ${ }^{23}$ We hope, although we have no evidence, that closure of such large foramens may prevent a paradoxical thromboembolus in the future. We are aware of two cases, one of which was reported in the medical literature, ${ }^{24}$ and one described in this report, where individuals with large patent foramen ovale had neurological decompression illness on one occasion (soon after diving), and thromboembolic stroke at a young age on another occasion (unrelated to diving). Transcatheter closure of patent foramen ovales and interatrial septal aneurysms with the Amplatzer septal occluders produced complete occlusion in six of seven patients in this study group without significant complications. All the patients have been able to return to diving, including the one with a small residual shunt. In that case, the shunt is no larger on contrast echocardiography than is present in a quarter of divers who do not get decompression illness.

This Amplatzer septal occluder in its current configuration is easy to implant and provides an effective non-surgical option for patients who have suffered neurological decompression illness related to a large patent foramen ovale. 
1 Hagen PT, Scholz DG, Edwards WD. Incidence and size of patent foramen ovale during the first 10 decades of life: an autopsy study

2 Thompson T, Evans W. Paradoxical embolism. OfM 1930; 23:135-50.

3 VanCamp G, Schulze D, Cosyns B, et al. Relation between patent foramen ovale and unexplained stroke. Am $\mathcal{F}$ Cardio 1993;71:596-8.

4 Haussman D, Mugge A, Daniel WG. Identification of patent foramen ovale permitting paradoxic embolism. $7 \mathrm{Am}$ Coll Cardiol 1995;26:1030-8.

5 Homma S, DiTullio MR, Sacco RL, et al. Characteristics of patent foramen ovale associated with cryptogenic stroke: a biplane transoesophageal echocardiographic study. Stroke 1994;25:582-6.

6 Cabanes L, Mas JL, Cohen A, et al. Atrial septal aneurysm and patent foramen ovale as risk factors for cryptogenic
stroke in patients less than 55 years of age: a study using transoesophageal echocardiography. Stroke 1993;24:1865tran

7 Schneider B, Zienkiewicz T, Jansen V, et al. Diagnosis of patent foramen ovale by transesophageal echocardiography and correlation with autopsy findings. $\mathrm{Am}$ f Cardio $1996 ; 77: 1202-9$

8 Moon RE, Camporesi EM, Kisslo JA. Patent foramen ovale and decompression sickness in divers. Lancet 1989;i:51314.

9 Guffi M, Bogousslavsky J, Jeanrenaud X, et al. Surgical prophylaxis of recurrent stroke in patients with patent foramen ovale: a pilot study. 7 Thorac Cardiovasc Surg 1996;112:260-3.

10 Bridges ND, Hellenbrand W, Latson L, et al. Transcatheter closure of patent foramen ovale after presumed paradoxical embolism. Circulation 1992; 86:1902-8.

11 Louie EK, Konstadt SN, Rao TL, et al. Transesophageal echocardiographic diagnosis of right to left shunting across the foramen ovale in adults without prior stroke. $7 \mathrm{Am} \mathrm{Coll}$ Cardiol 1993;21:1231-7.

12 Wilmshurst PT, Byrne JC, Webb-Pebloe MM. Relation between interatrial shunts and decompression sickness in divers. Lancet 1989;ii:1302-6.
13 Wilmshurst P, Davidson C, O'Connell G, et al. Role of cardiorespiratory abnormalities, smoking and dive character-
istics in the manifestations of neurological decompression istics in the manifestations of neurol
illness. Clin Sci 1994;86:297-303.

14 Mehan VK, Sideris EB, Meier B. Use of the reversed button device to treat an atrial septal aneurysm associated with a patent foramen ovale. Br Heart F 1995;74:563-5.

15 Ende DJ, Chopra PS, Rao PS. Transcatheter closure of atrial septal defect or patent foramen ovale for prevention of recurrence of paradoxic embolism. Am f Cardiol 1996;78:233-6.

16 Schenck MH, Sterba R, Foreman CK, et al. Improvement in noninvasive electrophysiologic findings in children after transcatheter atrial septal defect closure. Am f Cardiol 1995;76:695-8.

17 Sharafuddin MJA, Gu, X, Titus JL, et al. Transvenous closure of secundum atrial septal defects: preliminary results with a new self-expanding Nitinol prosthesis in a swine model. Circulation 1997;95:2162-8.

18 Bjornstad PG, Masura J, Thaulow E, et al. Interventional closure of atrial septal defects with the Amplatzer device: first clinical experience. Cardiology in the Young 1997;7:277-83.

19 Wilmshurst P, Walsh K, Morrison L. Transcatheter occlusion of foramen ovale with a button device after neurologision of foramen ovale with a button device after neurological decompression illness in professional divers. Lanc

20 Carminati M, Hausdorf $G$, Tynan $M$, et al. Initial clinical experience of transcatheter closure of secundum atrial septal defect with a septal occlusion system: a multicentre European study [abstract]. Eur Heart fु 1997;18(suppl): 136.

21 Hausdorf G, Schneider M, Franzbach B, et al. Transcatheter closure of secundum atrial septal defects with the atrial septal defect occlusion system (ASDOS): initial experience in children. Heart 1996;75:83-8.

22 Michel-Behnke I, Hagel KJ, Bauer J, et al. Transvenous closure of atrial septal defects in children with the DAS-angel wings device-alternative approach for selected patients [abstract]. Eur Heart $\mathcal{F}$ 1997;18(suppl): 136.

23 Knauth M, Ries S, Pohimann S, et al. Cohort study of multiple brain lesions in sport divers: role of a patent foramen tiple brain lesions in sport di
ovale. $B M \mathcal{F} 1997 ; 314: 701-5$.

24 Wilmshurst PT, Byrne JC, Webb-Pebloe MM. Neurological decompression sickness. Lancet 1989;i:731. 